\title{
Editorial
}

\section{Video Adaptation for Heterogeneous Environments}

\author{
Chia-Wen Lin, ${ }^{1}$ Yap-Peng Tan, ${ }^{2}$ Anthony Vetro, ${ }^{3}$ Alex Kot, ${ }^{2}$ and Ming-Ting Sun ${ }^{4}$ \\ ${ }^{1}$ Department of Electrical Engineering, National Tsing Hua University, Hsinchu 30013, Taiwan \\ ${ }^{2}$ School of Electrical and Electronic Engineering, Nanyang Technological University, Nanyang Avenue, Singapore 639798 \\ ${ }^{3}$ Mitsubishi Electric Research Laboratories, Cambridge, MA 02139, USA \\ ${ }^{4}$ Department of Electrical Engineering, University of Washington, Seattle, WA 98195, USA
}

Received 29 August 2007; Accepted 29 August 2007

Copyright (C) 2007 Chia-Wen Lin et al. This is an open access article distributed under the Creative Commons Attribution License, which permits unrestricted use, distribution, and reproduction in any medium, provided the original work is properly cited.

The explosive growth of compressed video streams and repositories which are accessible worldwide and the recent addition of new video-related standards, such as H.264/AVC, MPEG-7, and MPEG-21, have stimulated research for new technologies and applications in the area of multimedia architectures, processing, and networking. Current communication networks exhibit a wide range of capabilities, including various architectures, throughputs, and quality of service and protocols. The interconnection of different networks provides several advantages, but also poses major technical challenges. However, users employ heterogeneous videoenabled terminals such as computers, TVs, mobile phones, and personal digital assistants with a wide range of computational and display capabilities, energy resources, features, accessibilities, and user preferences. Such heterogeneity in networks and user devices has escalated the need for efficient and effective techniques for adapting compressed videos to better suit the different capabilities, constraints, and requirements of various transmission networks, applications, and end users. For instance, universal multimedia access (UMA) advocates the provision and adaptation of the same multimedia content for different networks, terminals, and user preferences.

Video adaptation is an emerging field that offers a rich body of knowledge and techniques for handling the huge variation of resource constraints (e.g., bandwidth, display capability, processing speed, and power consumption) and the large diversity of user tasks in pervasive media applications. Video adaptation may apply to individual or multiple video streams and call for different means depending on the objectives and requirements of adaptation. Transcoding, transmoding (cross-modality transcoding), scalable content representation, and content abstraction and summarization are popular means for video adaptation. In addition, video con- tent analysis and understanding, including low-level feature analysis and high-level semantics understanding, play an important role in video adaptation as essential video content can be better preserved.

Many research and development activities in industry and academia have been devoted to answering the challenges of making better use of video content across systems and applications of various kinds. This special issue aims at providing state-of-the-art developments in this flourishing and important research field. After a thorough review process, a total of 13 papers were selected, covering the topics of video adaptation tools, architecture design, performance analysis, complexity reduction, and real-world applications.

The first three papers cover the topic of video transcoding algorithms and their applications. Video transcoding is an operation of converting a video bit stream from one format into another format. It is an efficient means of achieving fine and dynamic video adaptation. The first paper by J. Xin et al., "efficient MPEG-2 to H.264/AVC transcoding of intra coded video," proposes a low-complexity transformdomain architecture and the corresponding mode-decision algorithms for transcoding intracoded video from MPEG2 to H.264/AVC format. Compared to the conventional pixel-domain approach, the proposed architecture reduces the transcoding complexity but incurs negligible or no loss in quality by performing direct coefficient conversion and mode decision in the transform domain. To further reduce the complexity, the paper also presents two fast mode decision algorithms, one evaluating the rate-distortion costs for a reduced set of modes decided by a simple cost function, and the other exploiting the strong temporal correlations between adjacent frames. The paper "efficient hybrid DCT-domain algorithm for video spatial downscaling," by N. Roma and L. Sousa, proposes a DCT-domain spatial 
downscaling transcoder for any arbitrary integer downscaling factor. The proposed algorithm reduces the computational cost while maintaining the visual quality by taking advantage of the scaling mechanism and by restricting the involved operations in order to avoid useless computations. In the paper "A multiple-window video embedding transcoder based on H.264/AVC standard," by C.-H. Li et al., a new transcoder is proposed to embed multiple foreground videos into one background video. The transcoding speed has been significantly improved by 25 times with negligible quality loss by using a combination of the slice group-based transcoding, reduced frame memory transcoding, and syntax level bypassing techniques. An improvement of up to $1.5 \mathrm{~dB}$ in PSNR is registered which is significant for multigeneration transcoding over the cascaded pixel domain transcoder.

Scalable video coding (SVC) is another powerful tool of adapting video content as it can provide different scaling options, such as temporal, spatial, and SNR scalability, where rate reduction by discarding enhancement layers of different scalability-type results in different kinds and/or levels of visual distortion depending on the content and bitrate. Two papers cover the topic of SVC-based video adaptation. The paper by W. Yang el al., "Scalable video coding with interlayer signal decorrelation techniques," proposes to improve the coding performance of the enhancement layers of SVC through efficient interlayer decorrelation techniques. This paper investigates, for both the open-loop and the closed-loop cases, two improved Laplacian pyramid structures for SVC that exploit the inherent redundancy of the underlying Laplacian pyramid with nonbiorthogonal filters by rendering the enhancement layer signal less correlated with the base layer. In "Content-aware scalabilitytype selection for rate adaptation of scalable video," E. Akyol et al. propose an objective function that quantifies flatness, blockiness, blurriness, and temporal jerkiness artifacts caused by rate reduction by spatial size, frame rate, and quantization parameter scaling. An adaptation method is proposed for choosing the best scaling type for each temporal segment that results in minimum visual distortion according to this objective function given the content type of temporal segments. Two subjective tests have been performed to validate the proposed procedure for content-aware selection of the best scalability type on soccer videos.

The next three papers address the problems of video adaptation in heterogeneous environments in terms of computation resources, perceptual quality, and channel bitrate. In "A complexity-aware video adaptation mechanism for live streaming systems," M.-T. Lu et al. propose a new video adaptation mechanism for live video streaming of multiple channels. This mechanism utilizes the complexity-distortion model to optimize globally through piecewise linear approximation in allocating the computational resource to each channel. A block-based complexity control method is also proposed to accurately control the computational resource of each channel on the live streaming server. The paper "An attention-information-based spatial adaptation framework for browsing videos via mobile devices," by $\mathrm{H}$. Li et al., presents an attention information-based spatial adaptation framework to address the problem of limited display sizes of mobile devices. The proposed framework includes two major parts: video content generation and video adaptation system. During video compression, the attention information in a video sequence is detected using an attention model and embedded into the compressed bitstream. The attention information is then employed to generate a bitstream of attention areas in each frame to adapt to the display sizes of mobile devices. Besides, an attention-biased QP adjustment scheme based on the attention information is proposed to regulate the output bitrate. The paper "Contentaware video adaptation under low bitrate constraint," by $\mathrm{M}$. $\mathrm{H}$. Hsiao et al., proposes a content-aware video adaptation method to retain visual quality under low-bitrate condition. The method first analyzes regions that are visually important based on the brightness, location, motion vector, and energy features derived in the compressed domain. Based on the analysis result, bit allocation is then performed, using additional motion features, at the frame level and, using a ratedistortion model, at the object level to achieve better visual quality.

Error robustness is crucial in video transport in error prone environments such as wireless networks. Four papers address this problem using different video adaptation techniques. The paper entitled "Comparison of error protection methods for audio-video broadcast over DVB-H," by M. Hannuksela et al., provides an excellent overview of audio-video transmission within the DVB-H environment, and analyzes the effectiveness of applying an unequal error protection scheme to compressed video within the context of such systems. The paper entitled "Transcoding based error-resilient video adaptation for $3 \mathrm{G}$ wireless networks," by S. Eminsoy et al. presents a video transcoding system that applies a combination of error resilience tools on the input compressed video streams to provide robust communications while regulating the output rates over a 3G W-CDMA wireless network. The paper also presents a new adaptive intra-refresh algorithm, which is responsive to the detected scene-activity inherently embedded into the video content and the reported time varying channel error conditions of the wireless network. In "Cross-layer design for video transmission over wireless rician slowfading channels using an adaptive multi-resolution modulation and coding scheme" by Y. Pei and Modestino, a scalable H.263+ video source coder is combined with unequal error protection across layers by employing different channel codes together with a multiresolution modulation schemes using nonuniform MPSK signal constellations. An adaptive joint source channel coding is also proposed and the results indicate good improvements in delivered video quality for specified channel conditions. The paper, "Multiple adaptations and content-adaptive FEC using parameterized RD model for embedded wavelet video," by Y.-H. Yu et al., presents a framework for performing multiple adaptations of wavelet-coded video whereby ratedistortion information is embedded in the video bitstream. The proposed framework also enables content-adaptive FEC protection. 
The final paper, "OLGA: a unified scalable framework for online gaming," by F. Morán et al. addresses an interesting application of video adaptation: online gaming. This paper describes how multiresolution representation and scalable coding can be exploited to adapt and deliver graphics content over heterogeneous networks and platforms. Some experimental results are presented to demonstrate the tradeoffs among the display quality, computational complexity, and bandwidth in rendering graphics content. The paper also discusses how content adaptation and load balancing can be achieved over a distributed content-delivery network.

\section{ACKNOWLEDGMENTS}

Guest editors are extremely grateful to all the authors who contributed their high quality papers to this special issue. They would like to thank all the reviewers who took time and consideration to assess the submitted manuscripts. They would also like to acknowledge the Editor-in-Chief and the Editorial Board members of the Journal on Advances in Signal Processing for their support of this special issue.

Chia-Wen Lin

Yap-Peng Tan

Anthony Vetro

Alex Kot

Ming-Ting Sun

Chia-Wen Lin received the M.S. and $\mathrm{Ph} . \mathrm{D}$. degrees in electrical engineering from National Tsing Hua University (NTHU), Hsinchu, Taiwan, in 1992 and 2000, respectively. He joined the Department of Electrical Engineering, NTHU, in August 2007, where he is presently an Associate Professor. Before that, he was with the Department of Computer Science and Information Engineering, National Chung Cheng Uni-

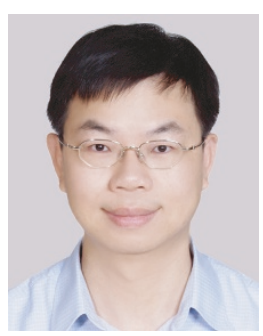
versity (CCU), Chiayi, Taiwan, during 2000-2007. Prior to joining academia, he worked for the Computer and Communications Research Laboratories (CCL), Industrial Technology Research Institute (ITRI), Hsinchu, Taiwan, during 1992-2000, where his final post was Section Manager. He has authored or coauthored over 70 technical papers. He holds a dozen patents with more pending. His research interests include video networking and video-content analysis. He is a Member of the Visual Signal Processing and Communications Technical Committee, the Multimedia Systems and Applications Technical Committee, and the Circuits and Systems Society of the IEEE. He is General Cochair of the First International Workshop on Multimedia Analysis and Processing (IMAP) held in Hawaii in August 2007. He is an Associate Editor of the Journal of Visual Communication and Image Representation. He has served as a Guest Coeditor of three special issues for EURASIP Journal on Advances in Signal Processing, Journal of Visual Communication and Image Representation, and Journal of Advances in Multimedia, respectively. He has also received several awards for his research work. He is a coauthor of the paper that won the Young Investigator Award at SPIE VCIP 2005. He received the Young Faculty Awards presented by CCU in 2005 and the Young Investigator Awards presented by National Science Council, Taiwan, in 2006.
Yap-Peng Tan received the B.S. degree from National Taiwan University, Taipei, Taiwan, in 1993, and the M.A. and Ph.D. degrees from Princeton University, Princeton, NJ, in 1995 and 1997, respectively, all in electrical engineering. He was the recipient of an IBM Graduate Fellowship from the IBM T. J. Watson Research Center, Yorktown Heights, NY, from 1995 to 1997, and was with Intel and Sharp Labs of America from 1997 to

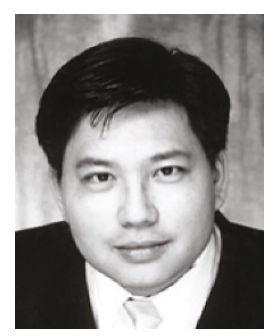
1999. In November 1999, he joined the School of Electrical and Electronic Engineering, Nanyang Technological University, Singapore, where he is presently an Associate Professor and Head of the Division of Information Engineering. His current research interests include image and video processing, content-based multimedia analysis, computer vision, and pattern recognition. He is the principal inventor/coinventor of 15 US patents in the areas of image and video processing. He is a Member of the IEEE Circuits and Systems Society's Technical Committee on Visual Signal Processing and Communications, an editorial board member of the EURASIP Journal on Advances in Signal Processing, EURASIP Journal on Image and Video Processing, and Advances in Multimedia.

Anthony Vetro received the B.S., M.S., and Ph.D. degrees in electrical engineering from Polytechnic University, Brooklyn, NY. He joined Mitsubishi Electric Research Labs, Cambridge, MA, in 1996, where he is currently a Group Manager responsible for multimedia research related to the video compression and transmission, information coding, and speech/audio processing. He has published more than 100 papers and

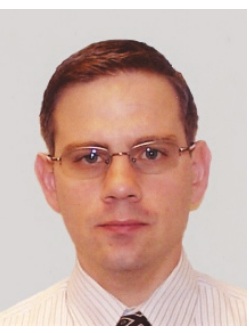
has been an active member of the MPEG and JVT standardization committees for several years. He serves on the program committee for various conferences and has held several editorial positions. $\mathrm{He}$ is currently an Associate Editor for IEEE Signal Processing Magazine, Chair-elect of the Technical Committee on Multimedia Signal Processing of the IEEE Signal Processing Society, and a member of the ICME Steering Committee. He is also a member of the Technical Committees on Visual Signal Processing and Communications and Multimedia Systems and Applications of the IEEE Circuits and Systems Society. He has been a Member of the Publications Committee of the IEEE Transactions on Consumer Electronics since 2002. Dr. Vetro has also received several awards for his work on transcoding, including the 2003 IEEE Circuits and Systems CSVT Transactions Best Paper Award and the 2002 Chester Sall Award.

Alex Kot received his B.S. degree in electrical engineering and MBA degree in Operational Management from the University of Rochester in Rochester, NY, and his Ph.D. degree in Electrical Engineering from the University of Rhode Island, Kingston, RI. He has been with the Nanyang Technological University, Singapore, since 1991. He headed the Division of Information Engineering at the School of Electrical and Elec-

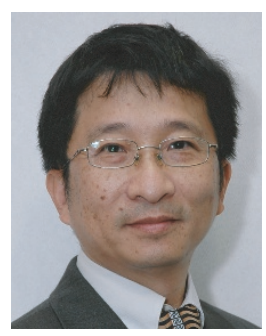
tronic Engineering for eight years. He is currently Professor and the Associate Chair Research (formally known as Vice Dean Research) for the School of Electrical and Electronic Engineering. He has published extensively in the areas of signal processing for communication, biometrics, data-hiding, and authentication. He served as an Associate Editor for the IEEE Transactions on Signal Processing, the IEEE Transactions on Circuits and Systems for Video 
Technology, and the IEEE Transactions on Circuits and Systems II. He is currently Associate Editor for the EURASIP Journal of Advances in Signal Processing and the IEEE Transactions on Circuits and Systems I. He is a Member of the Visual Signal Processing and Communication Technical Committee and the Image and Multidimensional Signal Processing Technical Committee. He has served the IEEE in various capacities such as the General Cochair for the 2004 IEEE International Conference on Image Processing (ICIP). He served as an IEEE Distinguished Lecturer and he is a Fellow of IEEE and IES.

Ming-Ting Sun received the B.S. degree from National Taiwan University, Taipei, Taiwan, in 1976, the M.S. degree from the University of Texas, Arlington, Tx, USA, in 1981, and the Ph.D. degree from the University of California, Los Angeles, in 1985, all in electrical engineering. He joined the University of Washington in August 1996 where he is a Professor. Before that, he was the Director of Video Signal Processing Research

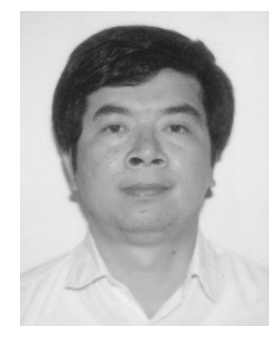
at Bellcore, where he led a group of researchers who participated in ITU and MPEG standards meetings, and provided significant contributions in the development of $\mathrm{H}$-series and MPEG video coding standards. His current research interests include video coding and other multimedia technologies. Professor Sun has been awarded 10 patents and has published more than 160 technical papers. He has coauthored 12 book chapters in the area of video and multimedia technology, and has coedited a book on Compressed Video over Networks. He has served in various leadership positions including the Chair of the IEEE CAS Standards Committee from 1988 to 1991, the Editor-in-Chief of IEEE Transactions on Circuits and Systems for Video Technology (TCSVT) from 1995 to 1997, the General Cochair of Visual Communication and Image Processing in 2000, a Distinguished Lecturer of the IEEE Circuits and Systems Society from 2000 to 2001, and the Editor-in-Chief of IEEE Transactions on Multimedia (TMM) from 2000 to 2001. He has received many awards including an Award of Excellence from Bellcore in 1987 for his work on digital subscriber line, an Award of Excellence from IEEE Standards Committee for the development of the IEEE IDCT standard, the TCSVT Best Paper Award in 1993, and the Golden Jubilee Medal from the IEEE CAS Society in 2000. Professor Sun is a Fellow of the IEEE. 